\title{
Experimental Robust Control of Structural Acoustic Radiation
}

\author{
David E. Cox* and Gary P. Gibbs ${ }^{\dagger}$ \\ NASA Langley Research Center \\ Hampton, VA 23661
}

\author{
Robert L. Clark $\ddagger$ \\ Dept. of Mechanical Engineering and Materials Science, Duke University \\ Durham, NC 27708-0302
}

\author{
Jeffrey S. Vipperman ${ }^{\S}$ \\ Dept. of Mechanical Engineering, University of Maine \\ Orono, ME 04469-5711
}

\begin{abstract}
$\underline{\text { Abstract }}$
This work addresses the design and application of robust controllers for structural acoustic control. Both simulation and experimental results are presented. $H_{\infty}$ and $\mu$-synthesis design methods were used to design feedback controllers which minimize power radiated from a panel while avoiding instability due to unmodeled dynamics. Specifically, highorder structural modes which couple strongly to the actuator-sensor path were poorly modeled. This model error was analytically bounded with an uncertainty model, which allowed controllers to be designed without artificial limits on control effort. It is found that robust control methods provide the control designer with physically meaningful parameters with which to tune control designs and can be very useful in determining limits of performance. Experimental results also showed, however, poor robustness properties for control designs with ad-hoc uncertainty models. The importance of quantifying and bounding model errors is discussed.

\footnotetext{
*Research Engineer, d.e.cox@larc.nasa.gov

$\dagger$ Research Engineer

$\ddagger$ Assistant Professor, Member AIAA

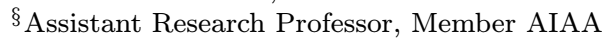

Copyright (C) 1998 by the American Institute of Aeronauthe copyright claimed herein for Governmental Purposes. All
} tics and Astronautics, Inc. No copyright is asserted in the United States under Title 17, U.S. Code. The U.S. Government has a royalty-free license to exercise all rights under other rights are reserved by the copyright owner.
\end{abstract}

\section{$\underline{\text { Introduction }}$}

The goal of this work was to apply modern robust control methods to a structural acoustic experiment, and investigate the advantages of robust control in this setting. Acoustic systems are often characterized by high modal density plants which extend well into and even beyond the controller's bandwidth. Modern control methods, such as LQR/LQG, have been successfully applied to the experimental control of high-order, structural acoustic systems (Vipperman and Clark, 1997). However, when the plant model is uncertain it is difficult to analytically determine the limits of achievable performance. Controllers must be designed with different levels of aggressiveness and experimentally tested to determine which will remain stable. Robust control offers the potential to determine, based on uncertainty models, if a controller is likely to destabilize the plant and to design optimal controllers which obtain performance objectives while retaining stability in the presence of model errors.

In modern control methods, such as LQR/LQG, the performance objective and system constraints are bundled into one cost function, for which an optimal system can be determined. These cost functions and the resulting controllers, however, assume accurate knowledge of the plant. In practice it is necessary to bound control effort or to invoke fictitious noise sources in order to obtain an optimal controller which is tolerant of model errors. Guide- 
lines have been developed, especially with regard to estimator noise statistics (Maciejowski, 1989) that help the control system designer find a suitable controller which not only meets the system's physical constraints, but also is relatively insensitive to model errors.

In robust control the goal is to incorporate the lack of model fidelity directly into the design procedure, and simultaneously optimize for performance and robustness. The mechanism for this involves the definition of an uncertainty model. Uncertainty models are viewed as magnitude bounds, up to which the plant is allowed to vary without affecting stability of the closed loop system. These bounds may be defined in a variety of ways, the simplest and most popular being unstructured uncertainty blocks. These uncertainties can be multiplicative at the plant input or output, or additive uncertainty around the plant. The multiplicative uncertainties define a level of unknown, but bounded coupling, among the system's inputs or outputs. Additive uncertainties are often used to bound the effect of dynamics which exist in the true system, but are not accounted for in the plant model.

This paper reviews the development of uncertainty models and motivates $H_{\infty} / \mu$-synthesis design methods. The presentation is not rigorous but rather tries to stress the physical interpretation and engineering significance of robustness properties. The work focuses on a structural acoustic experiment involving the transmission of sound through a rectangular aluminum plate. The plate model is finite and truncates a number of significant high-order modes. These high-order dynamics are then bounded by an additive uncertainty model. Determination of an appropriate uncertainty model, the control design, and experimental tests are covered in the sections which follow.

\section{$\underline{\text { Robust Stability }}$}

Robust control methods seek to find controllers that obtain required performance but are not sensitive to changes in the plant. In classical design methods robustness considerations can be handled heuristically by imposing design guidelines. For example, using a compensator to obtain pole-zero cancellation is not recommended for lightly damped systems. Gain and phase margins also provide good indicators of how sensitive a compensator is to model errors. However, in multiple-input multiple-output (MIMO) systems the designer has considerable freedom, and typically applies optimization techniques to obtain the control laws.
Robust controllers guarantee stability not only for a nominal plant model but also a set of possible plants defined by an uncertainty model. The simplest way to understand how the plant/uncertainty models interact is through a block diagram. Consider the block diagram shown in Figure 1. The nominal plant model, $P(s)$, has a controller $K(s)$, an uncertainty model $W_{a}(s)$, and the perturbation $\Delta(s)$.

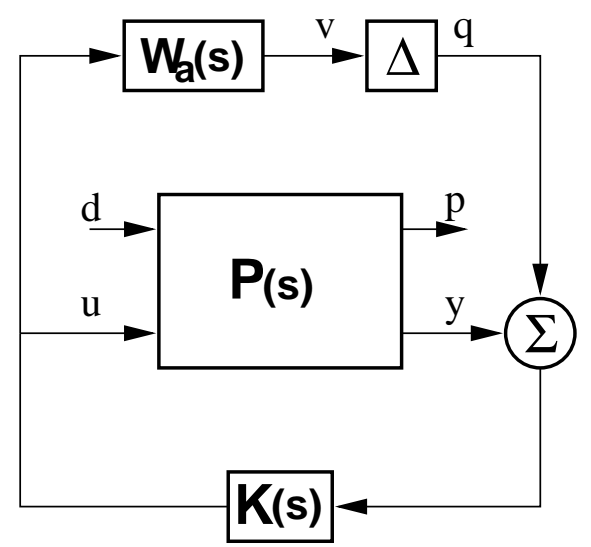

Figure 1: Nominal Plant with Additive Uncertainty Model

The perturbation $\Delta(s)$ defines the model variations, however it is not known explicitly. All that is known of the perturbation is that its infinity norm is bounded to be strictly less than unity, i.e. $\|\Delta(s)\|_{\infty}<1$. The infinity norm is sometimes referred to as an RMS gain norm. Both the input and output of $\Delta(s)$ are vectors, so gains across $\Delta(s)$ are defined as the ratio of vector two-norms, or RMS gains. The infinity norm is a single number which represents the largest RMS gain over all frequencies and over all input/output directions. The interpretation of signal and system norms is key to understanding robust control, and an excellent review can be found in Boyd and Barratt, (1991).

The uncertainty model $W_{a}(s)$ is used to scale the effects of $\Delta(s)$ and define bounds up to which the model may vary. This system represents a path in parallel with the plant model. It is often used to bound high-order dynamics of the true plant which are difficult to include accurately in the plant model. Although the phase response of this system is arbitrary its magnitude response must be scaled appropriately with the actual plant model.

A controller which has the property of robust stability is one which will remain stable for all $\Delta(s)$, and therefore all plant variations which are covered by the uncertainty model $W_{a}(s)$. The key to accomplishing this lies in the small gain theorem which 
states that a system will remain stable under closed loop conditions if

$$
\|L(j w)\|_{\infty}<1 \quad \forall \omega
$$

where $\|L(s)\|_{\infty}$ is the loop transfer function. To determine if a controller $K(s)$ has robust stability one simply examines the closed loop transfer function from the output of $\Delta$ to its input. This system, $P_{v q}$, can be viewed as one part of the loop gain, the remaining being the unknown perturbation set $\Delta(s)$. Although the explicit function $\Delta(s)$ is unknown, by definition it is bounded by $\|\Delta(s)\|_{\infty}<1$. From the triangle inequality we have,

$$
\|L(s)\|=\left\|P_{v q}(s) \Delta(s)\right\| \leq\left\|P_{v q}\right\| \cdot\|\Delta(s)\|
$$

where $\|\cdot\|$ denotes the maximum singular value at each frequency. This implies that if the norm of the transfer function $P_{v q}$ is less than one, the loop gain will be less than one, and thus the system will remain stable for all possible $\Delta(s)$. This is the condition for robust stability and can be interpreted as saying that the true system can have unmodeled dynamics of arbitrary order and arbitrary coupling up to the magnitude of $W_{a}(s)$, and the closed loop system will remain stable with controller $K(s)$.

\section{Robust Performance}

Satisfying the conditions for robust stability, however, says nothing about the performance of the system. Performance may degrade rapidly with small variations from the plant model even though the controller is still robust. Indeed, without a separate specification on performance the controller may do nothing at all. For unstable plants controllers can be designed based solely on robustness constraints, however, with stable plants the most robust controller typically has zero gain. This motivates modifying the robust control problem to include some measure of performance.

Our interest here is a disturbance rejection problem, which is to minimize the transfer function from a disturbance source to a performance output. This is the path $P_{p d}$, shown in Figure 1. Often in the literature the performance output is considered measurable, and hence corresponds to the output $y$. This has the advantage of allowing performance/robustness tradeoffs to be interpreted from the sensitivity and complementary sensitivity functions of the of the system. Here, however, the performance output is acoustic power radiated into an infinite half-space which can be predicted but not measured. The incorporation of this output into the system model is covered in detail in the next section.

The fully augmented system can be viewed as the standard three port model shown in Figure 2. The augmented plant $T$ has separate inputs for perturbations $q$, disturbance source $d$, and control signals $u$. It has separate outputs for perturbations $v$, performance $p$, and sensor signals $y$. The bounded system $\Delta(s)$ closes the upper loop and represents uncertainty in the plant model, and the feedback controller $K(s)$ closes the lower loop. Nominal performance is defined as $\left\|T_{p d}\right\|_{\infty}$, with $\mathrm{K}(\mathrm{s})$ closed but $\Delta(s)=0$. This is the the norm of the transfer function from disturbance to total radiated power when the system model is accurate, i.e. the nominal system model.

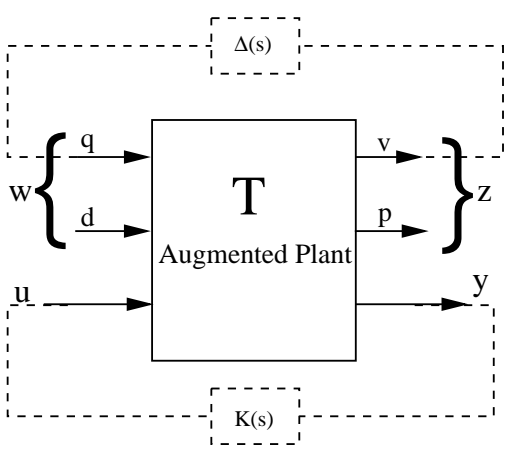

Figure 2: Generalized Plant Model, with grouped uncertainty and performance paths

To achieve robust performance it is necessary to simultaneously minimize the $T_{v q}$, the robustness transfer function and $T_{p d}$, the performance transfer function. The most straightforward approach is to consider all the respective inputs and outputs simultaneously, i.e. find $K(s)$ which minimizes

$$
\left\|T_{z w}\right\|_{\infty}=\left\|\begin{array}{cc}
T_{v q} & T_{v d} \\
T_{p q} & T_{p d}
\end{array}\right\|_{\infty}
$$

This is the optimal $H_{\infty}$ problem. Although it is not straightforward to directly find the optimal controller, numerical methods exist to find a suboptimal $K(s)$ which satisfies $T_{z w}<\gamma$ for some real constant $\gamma$ (Doyle et al., 1989). The optimal $H_{\infty}$ controller is then found through an iterative line-search which finds the lowest $\gamma$ for which a stabilizing controller exists.

Scaling is important to the $H_{\infty}$ problem. If $\gamma$ is greater than one the controller does not provide stability guarantees and either the performance weight or the uncertainty weight must be decreased. If $\gamma$ is much smaller than one it is likely that required 
performance specifications could be increased and robustness still satisfied.

Stacking the transfer functions into $T_{z w}$, as done in equation 3 provides a conservative guarantee on the underlying constraints. This is because it also bounds gains $T_{p q}$ and $T_{v d}$ which exist but have no robustness or performance interpretations. Robust performance is actually achieved if the performance constraint is met for all possible plant variations, ie

$$
\left\|T_{p d}\right\|_{\infty} \leq 1 \quad \forall \Delta(s) \in \Delta
$$

Clearly, it is not possible to test this condition for all possible $\Delta(s)$ in the allowed uncertainty structure $\Delta$. However, this condition can be guaranteed by assuring,

$$
\mu_{\hat{\Delta}}\left(T_{z w}\right)<1 \quad \forall \omega
$$

A proper development of $\mu$ is beyond the scope of this paper, but it is well documented and good presentations exist in Skogestad et al. (1996), and Balas et al. (1995). Conceptually, $\mu$ is similar to the infinity norm (although it does not satisfy the conditions of a norm) and is often called the structured singular value. It allows a singular value calculation to occur where the system of interest has structure, defined by $\Delta$. Although it is difficult to calculate $\mu$ directly, a reasonably tight upper bound is given by

$$
\mu_{\Delta}(M) \leq \operatorname{Inf}_{D(\omega) \in \mathcal{D}} \bar{\sigma}\left(D(\omega) M(j \omega) D^{-1}(\omega)\right)
$$

where $\mathcal{D}$ is the set of all $D$ which commute with $\Delta$. In fact, in the work presented here $\Delta$ has only two full blocks and the equality sign holds in equation (6). This function will yield a norm which is lower than the norm of $T_{z w}$, yet it can be shown to provide the same stability guarantees as the original. Calculation of $\mu$ is useful for problems with combined robustness and performance specifications, as well as problems that have more complex uncertainty structures.

The design of controllers which seek to optimize robust performance is called $\mu$-synthesis. Numerically, it involves placing an iterative loop around the $H_{\infty}$ solver which determines the best $D$ scaling for a given controller $K$, then reposes a weighted $H_{\infty}$ problem. This optimization, known as $D-K$ iterations, is not convex and not guaranteed to converge, however, it has been successfully applied in a large number of practical problems. The process puts $D$ $K$ iterations around the $\mathrm{H}_{\infty}$ solver's $\gamma$-iterations, which leads to a significant computational burden and adds states to the controller due to curve fitting of $D(\omega)$. The controllers described in this paper were all designed with $\mu$-synthesis, using routines available in the Matlab $\mu$-tools toolbox (Balas et al., 1995).

\section{$\underline{\text { Structural Acoustic Control }}$}

The system under study is an aluminum panel, instrumented with piezoelectric actuators and sensors, and mounted in a transmission-loss facility. The physical setup is discussed in more detail in the Experimental Structure section.

Although piezoelectric patches are the only sensors in the system, out-of-plane velocities from any point on the plate can be measured with a laser velocimeter. The laser system is configured to take data from a rectangular grid of points on the panel. These measurements must be made sequentially, and are not available for feedback. However, data from the laser is available for system identification, and the entire array of velocity points are outputs of the empirically derived model.

The goal is to attenuate acoustic transmission through a panel. Acoustic power can be predicted by considering the radiation efficiency of each of the structure's modes, and building a system which effectively transforms the modal velocities into radiation modes (Cunefare, 1991). This transformation can then be realized in a system model by building filters of arbitrary phase whose squared amplitudes match the radiation efficiency of each structural mode. Using this system, total radiated power can be derived from the model outputs and included as a performance output for control design (Baumann, 1991).

In the following work a slightly different method was employed. Following the development in of Elliot and Johnson (1993) radiation efficiencies were calculated based on a discretized form of Rayleigh's Integral. Total acoustic radiation was computed from an array of ideal monopole point sources. This has the advantage of being independent of the system model and only requiring a grid of velocity measurements to predict acoustic radiation. Specifically, the total power radiated from a baffled panel into a half-space, $\bar{P}$, is given by,

$$
\bar{P}=v^{H}(j w) R(j w) v(j w)
$$

where $v$ is an $m \times 1$ vector of panel velocities and $R$ is a real symmetric radiation matrix. The radiation matrix, $R$, can be determined analytically, however for use in control design it must be decomposed into a physically realizable system, $W_{r}$, such that

$$
R(j w)=W_{r}^{H}(j w) W_{r}(j w)
$$


Note that this relation leaves the phase of $W_{r}$ arbitrary and therefore the system $W_{r}(s)$ can be found through spectral factorization.

In this work the radiation efficiencies $R(j w)$ were not fit directly since for a grid of $n$ points this requires $n^{2}$ independent fits and leads to unreasonable system order. Instead the radiation matrix was factored into an ordered set of radiation modes via a singular value decomposition. The first few modes were curve fit, then used as basis functions for interpolation of the original full radiation matrix. This technique, termed Radiation Modal Expansion (RME), is detailed in Clark et al. (1997) and Gibbs et al. (1997) and based on the nesting property of radiation modes as described in Borgiotti (1994).

\section{System and Uncertainty Identification}

System Identification was performed using the observer identification methods available in the SOCIT matlab toolbox (Juang et al. 1992). This is a time domain approach which calculates Markov parameters from arbitrary input-output data, uses this data to form a Hankel matrix for the optimal observer and from this realizes a state space model for the system.

Inputs to the system were random pink noise which had been frequency weighted with a low pass filter in order to increase the excitation at low frequencies. This was found to be necessary because the piezo to piezo transfer functions tend to increase with frequency throughout the bandwidth of interest. Using pink noise gave output signals which were near the system's noise floor at low frequencies and yielded models with poor fidelity.

The system to be identified had 5 inputs: the disturbance speaker and four piezoelectric actuators, and 24 outputs: the grid of 20 velocity measurements and 4 piezoelectric sensors. It was not possible to obtain low-order models which had satisfactory accuracy. In part this was because the identification method attempts to preserve parts of the model which are significant to the input/output mapping. Similarly, doing balanced model reduction of high-order models tended to remove low frequency modes from the system model. Although this preserves a large portion of the system's response, it is at odds with the cost function, which is dominated by low frequency modes. Increasing the input signal at low frequencies improved the accuracy in these models, but doesn't increase their level relative to the high-order response.

A 200th order model for the system was realized which provided reasonable accuracy, both in matching frequency domain responses as taken experimen- tally from a spectrum analyzer and in matching time histories from input/output data sets not used in the identification process. The Nyquist rate of the digital system was $1800 \mathrm{~Hz}$, however, it was only of interest to control the system up to around $1000 \mathrm{~Hz}$. Therefore the 200th order model was decomposed into slow and fast portions, with the slow model containing dynamics up to $1000 \mathrm{~Hz}$ and the fast model above this. This yielded a model with 90 states which contained an accurate system model up to $1000 \mathrm{~Hz}$, and a very poor model above these frequencies.

To compensate for the system model inaccuracies at high frequencies a low-order uncertainty model was designed which would bound the high-order dynamics. Figure 3 shows the maximum and minimum singular values of $T_{y u}$, the plant model from actuator to sensor, in solid and dotted lines respectively. The dashed line in Figure 3 is the maximum singular values as calculated from the 4 by 4 transfer matrices in the experimentally determined spectrum, $T_{y u}$. The model response matches the spectra well up to about $1000 \mathrm{~Hz}$, where the model rolls off. An uncertainty model, $W_{a}(s)$ was defined which followed along the minimum singular values within the bandwidth of the model then increased to bound the unmodeled dynamics above $1000 \mathrm{~Hz}$. The gain of the uncertainty model is shown in Figure 3 with the dash-dot lines. The uncertainty model also ramped up below the first mode to bound model errors at low frequencies. No parametric uncertainties were included in the design model.

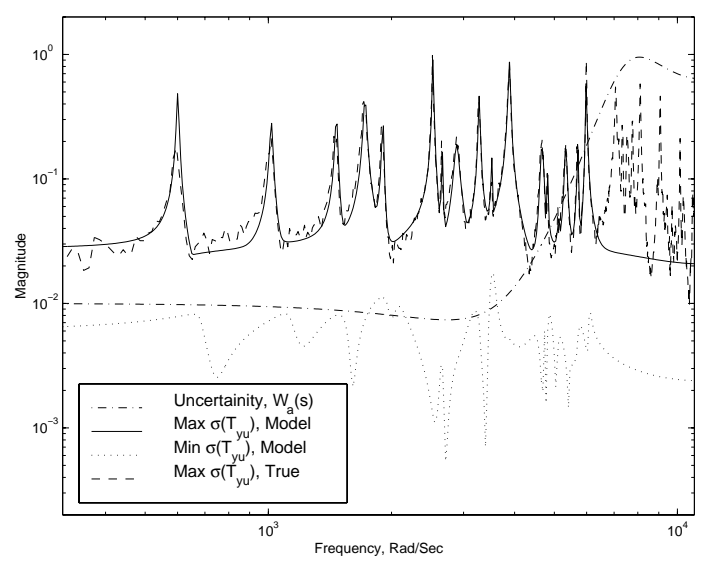

Figure 3: Max/Min $\sigma$ of model, Max $\sigma$ of True Plant, and Max $\sigma$ of uncertainty $W_{a}$ 


\section{Experimental Structure}

The structure under study is a 14" $\mathrm{x} 10$ " aluminum panel, clamped on all sides and mounted in the Transmission-Loss Facility at NASA Langley Research Center. Four ceramic piezoelectric patches are mounted on one side of the panel. These devices are configured as sensoriactuators, which can provide both actuation and sensing. An adaptive algorithm actively adjusts reference circuitry so that the piezoelectrics can accurately sense vibrations while simultaneously being used as actuators. Theory behind the sensoriactuator design can be found in Cole and Clark, (1994) and details of its implementation are described in Vipperman and Clark (1996).

Wideband disturbances are created with a loudspeaker. In a separate semi-anechoic room, microphone data was available and used for testing; however, final acoustic predictions are based on measurements from a scanning laser Doppler velocimeter. This information more accurately reflects the controllers design goals, and provides a better measurement of total radiated power. A schematic of the experimental setup is shown in Figure 4

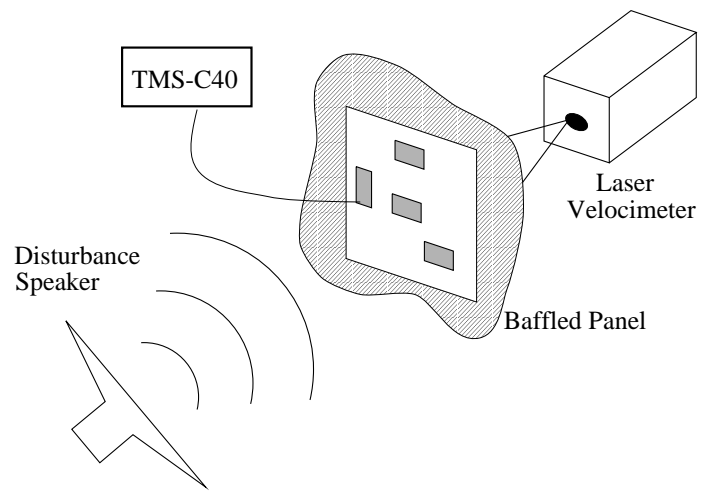

Figure 4: Experimental Setup

The real-time code was implemented in a TMSC40 digital signal processor. It implements a state space controller code for the 4-input 4-output sensor to actuator system and an LMS adaptive algorithm for the sensoriactuators' compensation. This adaptive compensation for the sensoriactuator circuitry requires an additional four channels of input and output voltages. For efficiency the controller only implements tridiagonal system matrices, so all controllers were transformed into modal coordinates prior to implementation. Care was taken to eliminate interchannel delays and minimize input-output delays, in order to approximate feed-through dynamics of the compensator.

Anti-aliasing filters were applied at $1600 \mathrm{~Hz}$, and smoothing filters were applied to the digital to ana$\log$ signals at $3200 \mathrm{~Hz}$. The system identification time records were recorded on the DSP and so contained these filters as part of the discrete plant model.

\section{Experimental Results}

Previous control experiments on this setup had shown that stable controllers used only a small range of the authority available from the piezoelectric actuators. Furthermore, although some noise was present on the measurements, anti-aliasing filters removed much of this and sensor signals were relatively clean. The control design process, therefore, did not include any penalty on control effort or any disturbance inputs representing sensor noise. The goals were simply to minimize the disturbance to performance path while providing a system which would remain stable for all dynamics bounded by the uncertainty model. This yielded a set of control laws which could be parameterized by a single design factor, the performance weight. Since the performance was the prediction of acoustically radiated power, no additional frequency weightings were applied.

Several controllers were designed, with various levels of performance weight. For each case $\mu$ synthesis was applied for four $D-K$ iterations, where further improvements in the value of $\mu$ were small. The controller parameters, intermediate and final values of $\mu$ and $\bar{\sigma}\left(T_{v q}\right)$ are shown in Table 1 . The columns I1 through I4 of this table show the results after each of the four $D-K$ iterations. These results predict that the final converged controllers of design $\mathrm{C} 1$ and $\mathrm{C} 2$ should be stable and $\mathrm{C} 3$ on the border of stability. The control designs $\mathrm{C} 4$ and $\mathrm{C} 5$ do not exhibit robust stability, and may be beyond the boundary of achievable performance.

When the designs were run experimentally, however, it was found that none of the five controllers were stable. The instability was not a high frequency mode, but stemmed from growing oscillations of modes well within the bandwidth of the system model. The cause of this instability is the relatively low uncertainty within this bandwidth, and the lack of other terms in the cost function which would restrain the control effort. As an attempt to counter this, flat multiplicative uncertainties were added at the input and output of the plant. Through experimental iteration, however, it was found that these uncertainties yielded controllers which were either overly conservative, or unstable. Given the system size this kind of iteration was very time consuming, taking several hours per controller on an Ultra- 


\begin{tabular}{||c||c|c|c|c|c||}
\hline \multirow{2}{*}{ Control } & Perf. & \multicolumn{4}{|c||}{ Robust Performance, $\mu$} \\
\cline { 3 - 6 } Law & Weight & I1 & I2 & I3 & I 4 \\
\cline { 3 - 6 } C1 & 5 & 4.76 & 1.02 & 0.75 & 0.74 \\
C2 & 7 & 7.14 & 1.41 & 0.95 & 0.90 \\
C3 & 10 & 9.52 & 1.94 & 1.24 & 1.10 \\
C4 & 12 & 11.89 & 2.33 & 1.42 & 1.23 \\
C5 & 15 & 14.28 & 3.00 & 1.65 & 1.40 \\
\hline & & \multicolumn{5}{|c||}{ Robust Stability, $\sigma\left(T_{v q}\right)$} \\
\cline { 3 - 6 } & & I1 & I2 & I3 & I4 \\
\cline { 3 - 6 } C1 & 5 & 0.15 & 0.48 & 0.73 & 0.73 \\
C2 & 7 & 0.14 & 0.50 & 0.76 & 0.88 \\
C3 & 10 & 0.23 & 0.57 & 0.80 & 1.08 \\
C4 & 12 & 0.20 & 0.59 & 0.85 & 1.20 \\
C5 & 15 & 0.29 & 0.60 & 0.95 & 1.36 \\
\hline
\end{tabular}

Table 1: Stablility and performance measures

Sparc. More significantly, however, this cut-and-try design method is exactly counter to the motivation for applying robust control. The objective of robust analysis is to have analytic predictions which indicate when a system's simulated performance is likely to be unobtainable in practice. Properly applied it should have physically motivated parameters which can be determined from system identification, and used to reduce the number of design/experiment iterations.

The optimal controller will use all available information about the plant to obtain performance and maintain stability against uncertainties. The $\mu$ values shown in Table 1 indicate that not much performance difference exists between the final controller and the next-to-last iteration. Viewing $\mu$ as a function of frequency, however, yields quite a different picture. Figures 5 and 6 show the open-loop performance, nominal performance, and robust performance $(\mu)$, for the third and fourth iterations of controller five, C5-I3 and C5-I4 respectively. In iteration 3, shown in Figure 5, the robust performance is degraded from the nominal as expected, but otherwise looks very similar. In iteration four, shown in Figure 6 the value of $\mu$ is nearly flat, and differs greatly from the nominal performance. This indicates a major change in the system output with perturbations. Controller C5-I4 is considered an improvement on C5-I3 because its peak value of $\mu$ over all frequency is is smaller. From a practical perspective, however, the third iteration produced a controller for which the system output does not vary as dramatically with perturbations, and using this

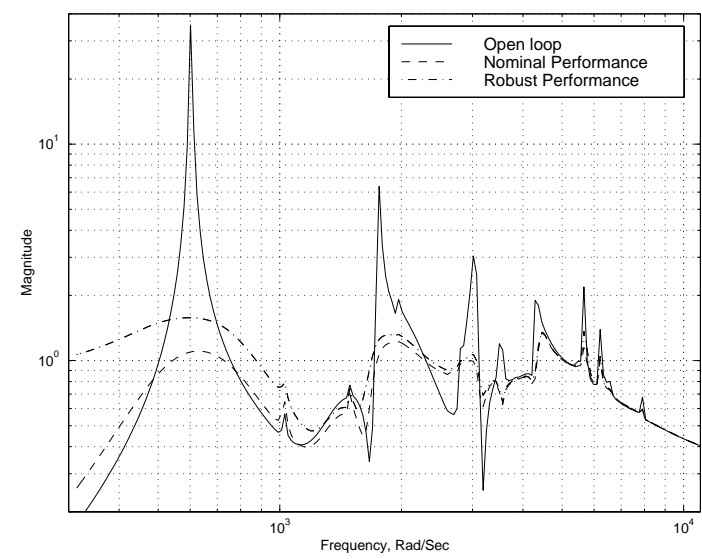

Figure 5: Open-Loop, Nominal Performance, and Robust Performance, with controller C5-I3

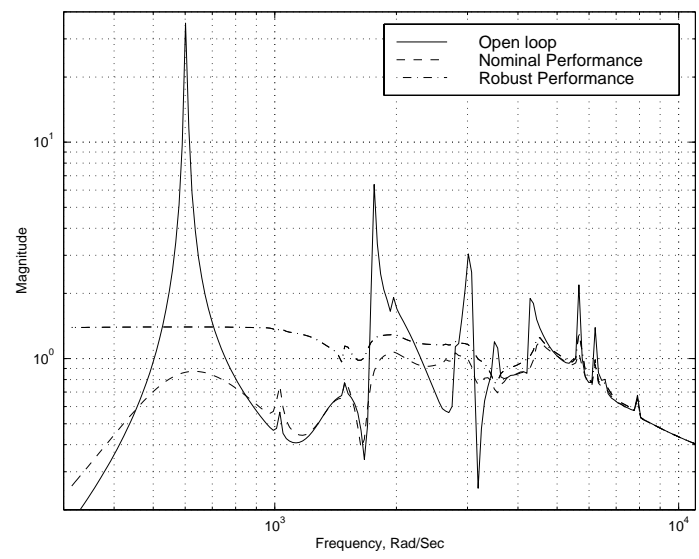

Figure 6: Open-Loop, Nominal Performance, and Robust Performance, with controller C5-I4

ad-hoc measure of robustness, this sub-optimal controller is clearly preferred.

The control laws in Table 1 were again implemented experimentally, this time using the second to last iteration. All of these controllers were stable and provided good performance, with controller C5-I3 providing the largest reduction. For this controller the total acoustic energy transmitted through the panel was calculated from a grid of 63 laser velocity measurements. This grid is twice the density of that used for prediction in the control law design. The speaker was driven by a pink noise source which was band-limited to $800 \mathrm{~Hz}$. Both open loop and closed loop measurements were taken, and a $7 \mathrm{db}$ reduction in total radiated power was achieved. The open and closed loop power predictions are shown in Figure 7 and the reduction in acoustic transmission over third octave bands is shown in Figure 8. 


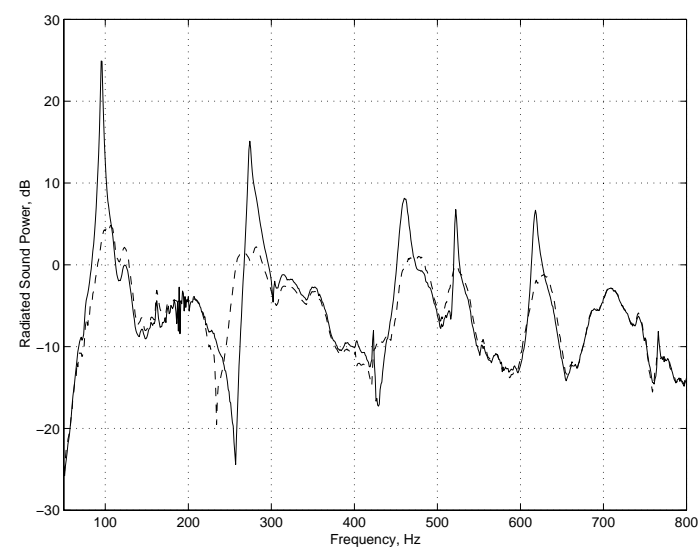

Figure 7: Experimental Open-Loop and Closed-loop (dashed) Performance with controller C5-I3

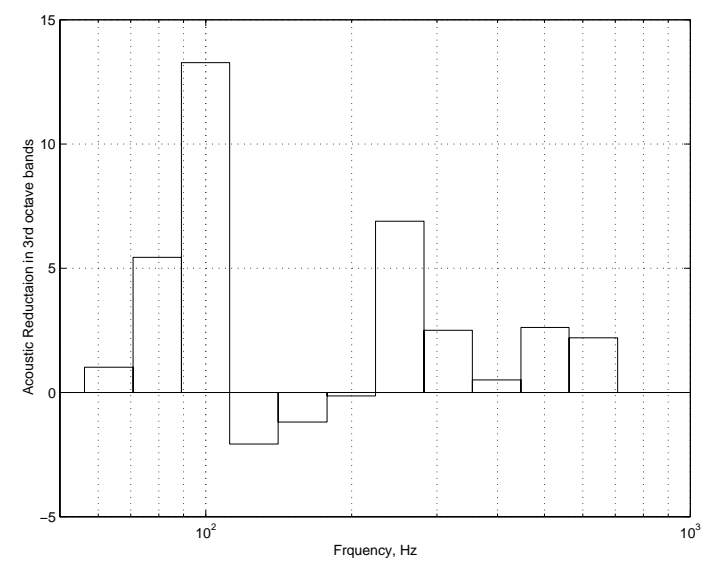

Figure 8: Reduction in radiated sound power over third octave bands with controller C5-I3

\section{$\underline{\text { Conclusions }}$}

This work examined the active control of a structural panel subject to acoustic disturbances. The objective of the control law was to reduce acoustic transmission through the panel, while maintaining stability in the presence of unmodeled dynamics. Piezoelectric adaptive sensoriactuators were employed as sensors and actuators in the system. The performance output of the system was the prediction of acoustic power radiation into a half-space. The identified system model contained a grid of velocity points on the panel, and were obtained from a laser velocimeter system. Predictions of radiated power were made by assuming each velocity point represented an acoustic monopole and fitting the radiation efficiency of these monopoles with realizable frequency domain functions.

The goal of this work was to examine the applica- bility of robust control methods to structural acoustic control experiments and gain insight from experimental implementation. Only frequency weighted uncertainties were considered, not parametric. It was found that uncertainty models are the critical parameter in determining the utility of robust control. Designs based on ad-hoc uncertainty models yielded poor performance, and more significantly had poor correlation between predictions of instability, based on large values of $\mu$, and actual instability seen in the lab.

The use of physically motivated uncertainty models, however, was found to yield good results. The explicit prediction of performance limitations is one of the most significant features of robust control and not available with other multivariable control methods. Robust control, despite its computational and theoretical complexities, provides the control designer with tuning parameters which are physically meaningful and can reduce experimental iterations on designs.

It was also found that using $\mu$ synthesis suboptimal controllers proved to be better in practice than their optimal counterparts. Partly this is because, in the work presented here, the two-norm is a better measure of desired performance than the infinity norm. A more significant observation, however, is that optimal controllers tended to be actively constrained by the uncertainty model and thus very dependent on the form of this model. Since uncertainty models are generally just overbounds based on physical observations, it is often not useful, and can be detrimental, to extensively optimize against these bounds.

\section{References}

[1] Balas, G.J., Doyle, J. D., Glover, K., Packard, A. K., and Smith, R., $\mu$-Analysis and Synthesis Toolbox, MUSYN Inc., Minneapolis, 1995.

[2] Baumann, W. T., W. R. Saunders, and H. H. Robertshaw, "Active suppression of acoustic radiation from impulsively excited structures," Journal of the Acoustical Society of America, 90(6), 3202-3208, (1991).

[3] Borgiotti, G. V., and Jones, K. E., 1994. "Frequency independence property of radiation spatial filters" Journal of the Acoustical Society of America, 96(6), pp. 3516-3524.

[4] Boyd, S., and C. Barratt, Linear Controller Design: Limits of Performance, Prentice Hall, Englewood Cliffs, NJ, (1991). 
[5] Clark, R. L., Saunders, W. R., and Gibbs, G.P., 1997. Adaptive Structures: Dynamics and Control, John Wiley \& Sons, New York.

[6] Cole, D. G., and Clark, R. L., 1994. Adaptive Compensation of Piezoelectric Sensoriactuators", Journal of Intelligent Material Systems and Structures 5, pp. 665-672.

[7] Cunefare, K. A., "The Minimum Multimodal Radiation Efficiency of Baffled Finite Beams," Journal of the Acoustical Society of America, 90(5), 2521-2529, (1991).

[8] Doyle, J.C., Glover, K., Khargonekar, P., and Francis, B., "State-space Solutions to Standard $\mathrm{H}_{2}$ and $\mathrm{H}_{\infty}$ Control Problems," IEEE Trans. on Auto Control, Vol.34, No.8, August 1989.

[9] Elliott, Sl J., and Johnson, M. E.1993 "Radiation modes and the active control of sound power", Journal of the Acoustical Society of America, 94(4) pp. 2194-2204.

[10] Gibbs, G. P., Clark, R. L., Cox D. E., and Vipperman, J. S., 1997. "Radiation Modal Expansion for Active Structural Acoustic Control", submitted to AIAA Journal.

[11] Juang, J. , Horta, L. G. and Phan, M. "System/observer/controller identification toolbox" NASA-TM-107566, (1992).

[12] Maciejowski,J. M., 1989 Multivariable Feedback Design, Addison Wesley, New York.

[13] Skogestad, S., and Postlethwaite, I., Multivariable Feedback Control, John Wiley \& Sons, New York, NY, (1996).

[14] Vipperman, J. S. and Clark, R. L., 1996. "Implementations of an Adaptive Peizoelectric Sensoriactuator", AIAA Journal, 34(10), pp. 21022109.

[15] Vipperman,J. S., and Clark, R. L., 1997. "Multivariable feedback active structural acoustic control with adaptive piezoelectric sensoriactuators", sumbitted to Journal of the Acoustical Society of America. 